\title{
TRIBUTEBANDS: IMITATION ALS ERFOLgSFAKTOR
}

\author{
Karl Menzel
}

»Wir bemühen uns wirklich sehr sorgfältig, die Lieder haargenau nachzuspielen. Wir wollen die Stücke nicht interpretieren und [nicht] unsere eigene Version daraus machen« (Colin Wilson, zit. n. Würfl 2004: 8).

Dieses Zitat des Bassisten der Australian Pink Floyd Show bringt das Motto der meisten Tributebands auf den Punkt: Angestrebt wird die möglichst exakte Kopie erfolgreicher Rock- oder Popacts. Anders als Coverbands bringen Tributebands nur die Musik eines einzigen Interpreten beziehungsweise einer einzigen Band auf die Bühne. ${ }^{1}$ Schon mit der Namensgebung wird der Bezug zum Original demonstriert, ist diese doch meist an einen Song- oder CD-Titel angelehnt oder basiert auf einer mehr oder weniger fantasievollen Umschreibung des Namens der imitierten Künstler: Black McSabbath, Gröne \& Meyer, Wobbie Rilliams, Still Collins, Bon Chauvi, Alanis Moreorless usw. Zum »haargenauen « Nachspielen der musikalischen Texturen kommt die Reproduktion ganzer Live-Kontexte, von der Imitation des entsprechenden Outfits bis hin zur Bühnenpräsentation und der dazugehörenden Rituale. So bieten Kissed Details diverser KISS-Shows:

»Optisch kaum vom Original Kiss zu unterscheiden, legen die vier ProfiVollblutmusiker [...] auf der Bühne richtig los. Mit unglaublichem Feuerwerk, brennendem Schriftzug und vielen weiteren Special-Effects wie Ace's brennender \& rauchender Gitarre, Gene, der Feuer und Blut spuckt, lassen sie ihren Auftritt zum unvergesslichen Erlebnis für das Publikum werden! Nicht nur die perfekt inszenierte Bühnenshow, sondern auch die vielen kleinen Details wie die originalen Musikinstrumente sind Garant für ein begeistertes

1 Anders als im angloamerikanischen Sprachraum wird in der deutschsprachigen Szene eine Trennung zwischen den Bezeichnungen »Coverband « und »Tributeband « nicht konsequent durchgehalten. Eine KISS-Tributeband kann genauso auch als KISS-Coverband bezeichnet werden. Der Systematik halber wird hier das angloamerikanische Modell übernommen und unterschieden zwischen Coverbands, die Stücke verschiedener Interpreten nachspielen, und Tributebands, die eine einzige Gruppe oder einen einzigen Interpreten nachahmen. 
Publikum« (www.kissfanshop.de/TributeBands/Kissed.htm; Zugriff: 5.10. 2005).

Bei der U2-Show der Hamburger Tributeband Zoo TV treten auch diverse »Gaststars« in Erscheinung:

»Die weiße Fahne (Sunday Bloody Sunday), der Zoo-Dollar-Regen (Desire), der legendäre Spot des Rattle \& Hum-Covers (Bullet The Blue Sky), Gastmusiker wie >Lou Reed (Satellite of Love), >Pavarotti< (Miss Sarajevo) oder Bläserunterstützung bei Angel of Harlem sowie diverse Kostümwechsel sorgten schon bei unzähligen Konzertbesuchern für echtes >U2-Feeling «!« (www.u2tribute.de/html/body_band.html; Zugriff: 20.12.2005).

Vergleichsweise bescheiden mutet es an, wenn der Udo-Jürgens-Nachahmer Andre Wolff verspricht, neben einer perfekten Stimm-Imitation auch »Mimik und Gestik wie das Original« zu bieten (www.andrewolff.de/udo.htm; Zugriff: 6.10.2005).

Von Veranstaltern und Publikum wird der Verzicht auf (künstlerische) Eigenständigkeit offenbar belohnt. Tributebands finden sich regelmäßig in den Programmen von Stadtfesten, Kneipenfestivals und vielen Musikclubs. Bands wie die Australian Pink Floyd Show, die Genesis-Doubles The Musical Box oder ABBA MANIA füllen mittlere und große Hallen, wobei viele $\mathrm{Zu}$ schauer bereit sind, für die Imitate Eintrittspreise von über dreißig Euro zu bezahlen.

\section{Die Szene}

Als Ursprung der Tributeszene werden vielfach die Elvis-Imitationswettbewerbe der 1970er Jahre ausgemacht, auf die eine kaum zu beziffernde Anzahl von Elvis-Nachahmern zurückgeht (Rubinowsky 1997). Ebenfalls in den 1970ern tauchten erste Beatlesduplikate auf. Gegen Ende des Jahrzehnts reüssierten in Nordamerika Bands, die auch aktuelle, noch existierende Formationen imitierten, z.B. Power Windows (Rush), Physical Graffiti (Led Zeppelin), Blushing Brides (Rolling Stones), Killer Queen (Queen), The Atomic Punks (Van Halen) (Kanaracus 2000 und 2001). Deren Erfolg brachte die Professionalisierung vieler Tributeprojekte und die Etablierung von Tributeshows als eigenständigem Unterhaltungssektor mit sich (Wells 1993; Hwang 2000; Boucher 2001; Tarlach 2002). Als Land mit ausgeprägter Tributekultur gilt Australien. In den späten 1980er und frühen 1990er Jahren tauchte dort eine größere Anzahl so genannter »Tributeshows« auf: Eine Madonna-Show, eine Doors-Show, eine Meat Loaf-Show mit Namen Fat Out 
of Hell, eine Blues Brothers-Show usw. Aber auch australische Bands wie Midnight Oil oder AC/DC wurden kopiert (Morrow 2005). Über die Landesgrenzen hinaus erfolgreich sind die australischen ABBA-Imitatoren Björn Again und die Australian Pink Floyd Show, die 2004 auch durch Deutschland tourte. Mittlerweile ist das Tributekonzept ein internationales Phänomen mit noch immer wachsendem Erfolg. Tributebands finden sich auch in Ländern wie Russland oder Japan (dort meist unter dem Begriff »Copy Bands «). Eine japanische Website verweist allein auf dreizehn Beatles-Copy Bands. ${ }^{2}$ Auf dem 2000er Edinburgh Festival spielte eine viel umjubelte Band namens The Japanese Beatles, die bei einigen Songs dann sogar von einem »Japanese Eric Clapton« begleitet wurde.

Die Session der »Fab Four « mit »Eric Clapton« zeigt aber auch die Grenzen im Authentizitätsstreben der meisten Tributebands: Viele Shows gleichen eher Greatest-Hits-Zusammenstellungen, als dass historische Konzertabläufe inszeniert werden. Beatles- oder ABBA-Tributebands bringen Songs auf die Bühne, die von den Originalbands nie live gespielt wurden, da sie zu einer Zeit aufgenommen wurden, als diese nur noch im Studio arbeiteten. Manch eine Doors-Tributeband überschreitet die meist nur gut eine Stunde dauernde Spielzeit der Konzerte des Originals um mehr als das Doppelte (Geary 2005: 484). KISS-Tributebands wie KISSIN' TIME bringen in Maskierung und Kostümierung Songs auch aus jenen Zeiten auf die Bühne, in denen KISS selbst Make-up und Verkleidung abgeschworen hatten (Interview mit Matthias Kreis und Thorsten Schütz vom 29.12.2005).

Bloßes Nischenphänomen im Tributegeschäft sind Bands, die sich vom reinen Kopieren lösen und stilistische Experimente oder eine ironische Annäherung an die Originale versuchen. So spielt Beatallica Stücke der Beatles im Metalsound von Metallica. Ein Projekt um das in Estland beheimatete Ensemble Rondellus versucht mit mittelalterlichen Instrumenten ein »early music tribute « an Black Sabbath und überträgt hierfür sogar deren Songtexte ins Lateinische (Tarlach 2002). Dread Zeppelin spielen Led ZeppelinSongs als Reggae-Version. Einen anderen stilistischen Transfer versuchen Hayseed Dixie mit der CD A Hillbilly Tribute To $A C / D C^{3}$, die sich mehr als 100.000fach verkaufte (Tarlach 2002). Ein in Los Angeles beheimateter »Mexican Elvis « mit Namen El Vez, laut Chris Kanaracus (2000) »a youngish guy with a coveted pompadour«, bringt Elvis-Songs mit spanischem und latein-

2 www.threeweb.ad.jp/ masappi/copyband.htm (Zugriff: 12.12.2005). Im Angebot der Seite sind auch Tributebands zu Toto, Emerson, Lake \& Palmer, Ronnie James Dio, Genesis, Queen sowie diverse Oldiebands.

3 Hayseed Dixie (2001). A Hillbilly Tribute To AC/DC. Western Beat/Dual Tone, 803020-1104-2. 
amerikanischem Einschlag: »)In the Ghetto becomes >En El Barrio<, and flamenco dancers flank the would-be icon« (ebd.).

Einen Eindruck vom Ausmaß des Tributephänomens geben einschlägige Websites. Auf Portalen wie www.coverthestars.de oder www.tributecity. com werden hunderte von Bands vorgestellt, Konzerttermine aufgelistet und Tipps zur Bandgründung gegeben; Besucher von Tributeshows erhalten Raum, sich auszutauschen und eigene Konzertkritiken zu veröffentlichen. Andere Seiten listen Tributebands auf, die allesamt demselben Originalact gewidmet sind. So finden sich z.B. unter www.kissfanshop.de/Tribute Bands/TributeBands.htm allein vierzig Fotos und Verweise auf KISS-Tributebands, www.crabsodyinblue.com/acdccoverbands.htm hat Links zu 109 AC/DC-Imitatoren zu bieten (Zugriff: 27.12.2005). Einige Booking-Agenturen und Veranstalter machen ihr Geschäft vorrangig oder ausschließlich mit Tributebands. Im Limelight Club (www.crewe-limelight.co.uk) im mittelenglischen Crewe, der nahezu täglich Livemusik im Angebot hat, werden Formationen wie Wishbone Ash schon explizit als »Originalbands « angekündigt, damit sie nicht mit einem der vielen Duplikate im Programm verwechselt werden. In den Niederlanden wird alljährlich die beste Tributeband gewählt und mit dem eigens ins Leben gerufenen Dutch Undercover-Award ausgezeichnet. Kultstatus haben auch die regelmäßig im renommierten Rotterdamer Musikclub Ahoy stattfindenden Tribute Band Nights. Die Veranstalter werben damit, »the best bands of the world in one show« zu bieten, und führen ihre Besucher in eine imaginäre Musikwelt, die selbst zum Kunstobjekt geworden ist (www.tributebandnight.nl).

\section{Tributebands in Deutschland}

Der erste deutsche Tributeact mit überregionaler Bedeutung war sicherlich die Beatles Revival Band aus Frankfurt. Sie wurde 1976 gegründet und hat die Lebenszeit der Original-Beatles nunmehr schon um ein Vielfaches überschritten: 2006 feiert sie ihr dreißigjähriges Bestehen und hat bisher mehr als 4000 Konzerte gegeben. Hauptprogramm ist eine Magical History Tour, laut Band

»Eine Zeitreise durch die sSixties` - Von den Anfängen im Hamburger Top Ten bis zum letzten Konzert der Fab Four auf den Dach der Emi Studios in London. Alles im original Outfit der jeweiligen Ära. Die ultimative BeatlesShow « (www.beatlesrevivalband.info/brb2/html/shows.html; Zugriff: 7.10. 2004). 
Neben dieser History Tour wird noch ein Beatles meets Classic betiteltes Programm gegeben, welches »Unterhaltung, Kultur und Überraschung zugleich « darstellen soll - dabei handelt es sich, laut Band, um eine »beeindruckende Kombination legendärer Beatles-Songs mit der klassischen Musikbegleitung eines Sinfonie-Orchesters « (ebd.). Wohl als Reminiszenz an neuere Entwicklungen ist mittlerweile auch eine Beatles-Unplugged-Show im Angebot.

Die folgende Auflistung zeigt die >Top Ten< der von deutschen Tributebands imitierten Bands/Interpreten: ${ }^{4}$

$\begin{array}{lc}\text { AC/DC } & 51 \\ \text { Elvis } & 11 \\ \text { The Beatles } & 10 \\ \text { Pink Floyd } & 8 \\ \text { Robbie Williams } & 8 \\ \text { ABBA } & 7 \\ \text { Rolling Stones } & 7 \\ \text { U2 } & 6 \\ \text { Bon Jovi } & 5 \\ \text { KISS } & 4\end{array}$

Auch von deutschsprachigen Künstlern gibt es Tributebands: von den Toten Hosen ( 5 kleine Jägermeister; Die Toten Hosen Coverband) über Udo Lindenberg (Johnny Controlletti und das Katastrophenorchester), Marius MüllerWesternhagen (Pfefferminz, Halle-Luja, Westernhagen-Revivalshow) und Rammstein (Stammheim, Feuerengel) bis hin zu Udo Jürgens (André Wolff, SahneMixx). Ein Projekt um den Kasseler Stimmenimitator Fred Niebert bringt eine komplette »Deutschrock-Legenden«-Tributeshow auf die Bühne und hat dabei Lindenberg, Westernhagen, Grönemeyer, Peter Maffay und Nena im Programm (www.deutschrock-coverbands.de).

\section{Tributebands und >Originales}

Die Imitation von Musik, Show, Outfit, Gestik und Mimik bringt urheber- und persönlichkeitsrechtliche Konflikte mit sich. Die juristische Lage ist nicht abschließend geklärt: „While publicity rights are usually applied to cases involving advertising, a strong case exists to apply these same rights to

4 Grundlage war deren Internetpräsenz (Zugriff: 20.10.2005). Tributebands ohne eigene Homepage wurden deshalb nicht erfasst. 
disputes regarding the commercial profits made by tribute bands « (Geary 2005: 482). Die Reaktionen von Original-Künstlern bzw. deren Rechteinhabern sind unterschiedlich. So wurde der Beatles Revival Band in ihren Anfangstagen mit einer Unterlassungsklage seitens des Beatles-Verlags gedroht. Drummer Ringo Star versuchte es mit finanziellem Anreiz und bot dem Vernehmen nach 500.000 DM, wenn die Band ihre Aktivitäten einstelle. Krissi Geary (2005: 489ff.) listet Verfahren vor US-amerikanischen Gerichten auf, in denen gegen Tributebands oder vergleichbare Imitationsshows geklagt wurde. Aktuelles Beispiel für juristische Drohgebärden ist die von Sony geführte Unterlassungsklage gegen Beatallica (Roth 2005; Dambeck 2005).

Eine gegensätzliche Verhaltensweise legte Pink-Floyd-Gitarrist David Gilmore an den Tag. Er war von der Australian Pink Floyd Show derart angetan, dass er mehrmals deren Konzerte besuchte und die Band exklusiv zu seinem 50. Geburtstag in den Londoner Earl's Court einlud - wo sie im Anschluss an ihre Show noch mit Mitgliedern des Original-Floyd-Lineups und anderen prominenten Musikern jammen konnte. Besonders aufgeschlossen für seine Imitatoren zeigt sich auch Phil Collins. Auf seiner Homepage weist er direkt auf die Tourdaten von Tributebands hin, darunter auch die einiger deutscher Gruppen. Vor seinem Konzert am 23. Juni 2004 in der Stuttgarter Schleyerhalle empfing er die Bruchsaler Collins-Tributeband Phil zum Plausch unter Musikern. Dem Phil-Sänger Jürgen Mayer wurde die Ehre zuteil, während des Konzerts - und auf Großleinwand übertragen - eine Refrainzeile des Songs "Sussudio « zusammen mit Collins zu singen. ${ }^{5}$

Im Allgemeinen scheint die Ansicht vorzuherrschen, dass Tributeacts für die kopierten Originale von finanziellem Nutzen sind, oder wie Geoff Boucher (2001) formuliert: »[A] Bruce Springsteen tribute act is [...] making money for the Boss. « Veranstalter von Tributekonzerten zahlen Urhebergebühren, die den Original-Künstlern zugute kommen. Auch verspricht man sich einen Gewinn durch die möglicherweise angekurbelte Nachfrage nach originalen Tonträgern oder DVDs (Wells 1993). Werner Leonard (Interview vom 7.11.2005), Initiator von ABBA Gold und ABBA MANIA, sieht seine Shows somit auch als Werbeträger für ABBA selbst, da es hierdurch »immer neue Fans für die ABBA-Musik gibt«. Einige Bands gehen soweit, »offizielle« Tributebands zu benennen. Die KISS-Tributeband Hotter Than Hell wurde gar von KISS-Frontman Paul Stanley selbst initiiert, der seinen früheren Gitarrentechniker Tony Sabetta mit einbezog und die Band auch als Opener für

5 Der Link zu Collins' Homepage lautet: www.philcollins.co.uk/Tribute.htm. Collins begrüßte seinen Imitator ironisch mit »Are you me? «, gab allerdings zu, noch nie eine Collins-Tributeband live gesehen zu haben (www.phil-online.de; Zugriff: 12.12.2005). 
eigene Konzerte engagierte (Kanaracus 2000). Unter KISS-Tributemusikern hält sich das Gerücht, dass der Entschluss von KISS, 1996 nach einer längeren Make-up-freien Zeit wieder in Originalbesetzung und mit Schminke und Kostümen aufzutreten, auch auf den andauernden Publikumserfolg der Tributebands zurückzuführen ist (Interview mit Thorsten Schütz vom 29.12. 2005). Über einen offiziell abgesegneten Status verfügen auch die MetallicaImitatoren Battery und die Aerosmith-Tributeband Draw The Line, die bei Auftritten Merchandising-Artikel der Originalbands verkaufen und Originallogos und -slogans benutzen dürfen (Kanaracus 2000; Geary 2005: 486f.). Die Tributeband The Musical Box ließ sich ihre Darbietung von »Genesis on Tour 1972-75« durch die Original-Genesis ebenfalls förmlich absegnen (Pendzich 2005).

Gelegentlich kommt es zum >Crossover « zwischen der Tribute- und der Originalband-Szene. So ist der ehemalige Boston-Leadsinger Brad Delp mittlerweile Mitglied der relativ erfolgreichen amerikanischen Beatles-Tributeband Beatlejuice. Und der zeitweilige Whitesnake-Gitarrist Amos Sanfilippo spielt in einer Van-Halen-Tributeband namens Diver Down (Kanaracus 2001). Andersherum gibt es mit dem Fall des Sängers Tim »Ripper« Owens zumindest ein Beispiel dafür, dass eine Original-Band, hier Judas Priest, einen Nachfolger für den ausgestiegenen Vokalisten aus einer sich ihr widmenden Tributeband rekrutierte. Dem Regisseur Stephen Herek erschien der wundersame Aufstieg Owens' spektakulär genug, um inn zum Thema eines Spielfilms (Rock Star, 2001) zu machen.

\section{Rollentausch}

Für die Dauer des Auftritts schlüpfen Tributemusiker in die Rolle der gecoverten Interpreten. Eigenständige künstlerische Kreativität ist dabei im Normalfall nicht gefragt, wohl aber das minutiöse Imitieren musikalischer Texturen. Um der Authentizität Willen werden vielfach Live-Versionen herangezogen (vgl. Groebbels 2004). Die Aneignung der Originalparts geschieht entweder durch Heraushören der Vokal- und Instrumentalstimmen von Tonträgern oder Videomitschnitten. Leonard (Interview vom 7.11.2005) beschreibt, wie z.B. Filtertechniken genutzt werden, um Details der Gesangsparts von ABBA-Aufnahmen zu extrahieren. Oder man greift auf Transkriptionen zurück, die für eine Vielzahl bekannter Stücke erhältlich sind. Eine weitere Möglichkeit, an brauchbare Spielvorlagen zu kommen, bieten die für Sequenzer-Anwendungen konzipierten Standard-MIDI-Files, die meist komplette Band-Arrangements enthalten (Menzel 2005: 121ff.). 
Akribische Arbeit erfordert auch die technische Umsetzung: Der NeilYoung-Imitator Dennis Neil beschreibt, wie er mehrere Tausend Dollar dafür ausgab, eine originale Gretsch White Falcon-Gitarre und eine schwarze Les Paul mit Bigsby-Tremolo und verchromter Hardware zu bekommen, also die Gitarren, die von Young in den 1970er Jahren gespielt wurden. Bei der Zusammenstellung des Instrumentariums kontaktierte er Neil Youngs Techniker über das Internet und ließ sich mit Tipps über dessen Equipment und die genauen Modifikationen der Verstärkeranlage versorgen (Simons 2001). Für ABBA Gold wurde die sternförmige Gitarre, eines der Markenzeichen von Björn Ulvaeus, »richtig mit CAD-System vermessen« und nachgebaut (Interview mit Leonard vom 7.11.2005). Matthias Kreis und Thorsten Schütz von KISSIN' TIME beschreiben, wie sie besonders in der Anfangszeit immer wieder damit beschäftigt waren, ihren Gitarren den originalen KISS-Look zu geben:

»Da gab es kaum einen Monat, wo mal nicht eine Gitarre auf dem Seziertisch lag. Und auch heute noch: Wenn der Paul Stanley mal wieder 'ne neue Gitarre hat oder der Gene wieder einen anderen Bass, dann müssen auch wir wieder ran« (Interview mit Kreis und Schütz vom 29.12.2005).

Eine Alternative zu Eigenbau oder Modifikation bieten so genannte »ArtistSignature-Instrumente «, mit denen führende Hersteller Nachbauten der von Stars gespielten Instrumente liefern. Diese sind mitunter im »Relic-Look« auf alt getrimmt und versprechen, neben der Optik, auch spezifische Soundcharakteristika und Spielbedingungen zu ermöglichen. ${ }^{6}$ Auf der Soundebene bietet digitale Sampling- oder Verstärkertechnologie zudem die Möglichkeit, bekannte Sounds originalgetreu zu emulieren und die hierzu erforderlichen Soundeinstellungen als »Presets « über das Internet zu beziehen. ${ }^{7}$ Line6, ein

6 Fender liefert z.B. seine Stratocaster u.a. in den Versionen von Eric Clapton, Jeff Beck, Yngwie Malmsteen, Buddy Guy, Mark Knopfler, Robert Cray und Stevie Ray Vaughan aus (CDs der Stars werden beim Kauf mitunter mitgeliefert).

7 Soundpresets sind digital gespeicherte Algorithmen, die z.B. berechnen, wie sich das Klangverhalten eines Synthesizers oder Samplers unter bestimmten Umständen verhalten würde und die dies mittels Digital/Analogwandlung hörbar machen. Die meisten Hersteller bieten Internetseiten, auf denen sich die erforderlichen Sounds herunterladen lassen. Beim »Amp-Modelling « wird der Klang bestimmter Gitarrenverstärker und Lautsprecherboxen digital berechnet. Eines der ersten Geräte, mit dem dieses Verfahren im größeren Stil vermarkt wurde, war der für das Recording mit dem PC konzipierte, von Line6 entwickelte Pod. Der Pod bietet eine Auswahl aus 24 Verstärkermodellen, die mit verschieden Lautsprechertypen, Effekten und Einstellungen zur Raumsimulation kombiniert werden können. Mittlerweile ist dieses System aber auch in bühnentaugliche Verstärker eingebunden. Somit ist es möglich, so vollkommen unterschiedliche Sounds wie die von Carlos Santana, Mark Knopfler, Jimi Hendrix oder Joe Satri- 
Marktführer der »Amp Modelling «-Technologie, tritt bezeichnenderweise auch als einer der Hauptwerbepartner des Tributeband-Portals Tribute City auf.

Aufwändiger als der musikalische Teil erweist sich oftmals die Zusammenstellung des vorgegebenen Outfits. Hierzu noch einmal der Neil YoungImitator Dennis Neil:

"You've got to get the artist's look and sound together. For us that meant to get to a head-to-toe Neil look going. [...] So I hit up many of the local costume and wig shops and Salvation Army stores and found everything I needed to do the trick. Since I look nothing like Neil, it takes quite a makeover to get ready for our performances « (Dennis Neil, zit. n. Simons 2001).

Geary (2005: 483) berichtet von einer Beatles-Tributeband, die den Schneider der Beatles kontaktierte, und von ZZ-Top-Doubles, deren Mitglieder jahrelang auf das Rasieren verzichteten, um sich auch in punkto Bartwuchs den Originalen anzugleichen. Der Drummer Rolo Sandoval, der über zwei Jahrzehnte in gut einem Dutzend Beatles-Tributebands mitwirkte, ließ sich eine spezielle Kunststoffnase für 300 Dollar anfertigen, um dem von inm verkörperten Ringo Starr ähnlicher zu sein (Boucher 2001). Schütz beschreibt die Mühen bei der Verwandlung in Paul Stanley von KISS:

»Das ist immer eine ziemlich große Aktion. Wir hatten allein schon Probleme, überhaupt die Schuhe zu kriegen. Ich bin auf Flohmärkte gefahren, Ebay, alles abgegrast. Selbst wenn du echte Plateau-Platformboots überhaupt kriegst, dann sind sie immer noch nicht hoch genug, wie sie für KISS sein müssen. Dann gibst du sie zum Schuster, lässt sie hoch machen, sie müssen ja auch stabil sein. Du darfst ja nicht auf der Bühne rumstolzieren, Spagatsprünge machen und dann bricht dir der Absatz ab. Da kommen Federstahlbleche rein. Einen Teil der Kostüme lassen wir nähen, aber z.B. die Ritterrüstung vom Andy, die habe ich halt selbst gemacht. Und dann wird Leder gekauft, Leder ist ja auch nicht gerade billig. Und die Ritterrüstung, die ist auch nicht aus Metall, die ist aus Plexiglas. [...] Wir haben uns die echte KISS-Schminke aus den Staaten mitbringen lassen, mit der wir dann dummerweise überhaupt nicht klargekommen sind. Dann haben wir Schminke über die Theaterdrogerie bezogen. Es hat Jahre gedauert, wo ich das Versuchskaninchen war. Mir ist manchmal während der Show ab dem zweiten Lied die Schminke weggelaufen. Jetzt habe ich wieder Kontakt zur Maskenbildnerin vom Thea-

ani mit nur einem Verstärker zu erzielen. Noch einfacher wird dies, wenn man die ebenfalls von Line6 entwickelte Gitarre Variax benutzt, die das Klangverhalten gängiger Gitarrenmodelle mittels integriertem Mikrochip nachahmt. Eine von Line6 bereitgestellte Website zum »ToneTransfer (www.customtone. com) bietet exakte Soundeinstellungen, darunter z.B. von Mark Knopflers Solo in "Sultans of Swing « oder Jimi Hendrix' Solo in »Hey Joe«. 
ter aufgenommen, weil Paul Stanley Haare auf der Brust hat und ich nicht. Das war das letzte Tüpfelchen auf dem i, wo ich gedacht hab', das müsste ich jetzt auch haben« (Interview mit Kreis und Schütz vom 29.12.2005).

Ungefähr zweieinhalb Stunden benötigt Schütz für die Verwandlungsprozedur. Vorher muss noch die Bühnenanlage aufgebaut und die Pyrotechnik installiert werden.

\section{Only in it for the Money? Die ökonomische Seite}

"And after years of playing for pennies in original acts, local musicians are finding a ready-made fan base that's willing to pay money to hear them pay tribute to Rush, KISS and even the BoDeans« (Tarlach 2002).

Auf die Frage "What do you think of the state of the current rock music scene? Is it easy to get gigs? « antwortet der schottische Eddy van HalenImitator Paul Logue geradeheraus: »Tribute - YES, Original - NO!« (zit. n. Ritchie 2003). Der Wechsel ins Tributegeschäft kann sich auszahlen. So entstand die amerikanische Jimi-Hendrix-Tributeband Wild Blue Angels aus einer Bluesformation. Mitauslöser für den Wandel war die Frustration über eine schlechte Platzierung bei einem Open-Air-Festival und der Erfolg nach dem spontanen Entschluss, das ursprünglich geplante Programm durch eine Hendrix-Show zu ersetzen. Seitdem absolviert die Band bis zu fünfzehn Auftritte pro Monat (Kanaracus 2000). In einem aktuellen Bericht über den Einfluss des Tributeband-Phänomens auf die australische Live-Szene zeigt Guy Morrow (2005), dass sich das Prinzip Imitation sowohl im Hinblick auf die Auftrittsmöglichkeiten als auch in Bezug auf die Höhe der Gagen auszahlt: Findet eines der ohnehin eher seltenen Konzerte statt, bei dem Eigenkompositionen präsentiert werden, so sind pro Abend meist drei verschiedene Bands zu hören. Festgagen werden nicht gezahlt, es bleiben allein die Eintrittsgelder. Da diese Konzerte meist in kleineren Auftrittsorten stattfinden, ist die Gage entsprechend gering. Morrow rechnet für die Szene in Australien vor, dass selbst im Idealfall bei einer Zuschauerkapazität von 120 Personen und einem Eintritt von acht australischen Dollar pro Band nur 320 \$ übrig bleiben. Hiervon müssen noch sämtliche Kosten abgezogen werden. Morrow geht aber im Allgemeinen von wesentlich geringeren Einnahmen aus, da die Konzerte selten ausverkauft sind und darüber hinaus viele Besucher aus dem Umfeld der Bands keinen Eintritt bezahlen. "Paying to play« gehört deshalb, laut Morrow, für viele dieser Formationen zum Alltag. 
Tribute- und Coverbands hingegen werden in Australien deutlich häufiger gebucht und spielen nahezu ausschließlich gegen Festgage, die sich je nach Auftrittsort auf bis zu $2000 \$$ (immerhin gut $1200 €$ ) belaufen kann. Viele australische Musiker spielen deshalb in Tribute- oder Coverbands, um Projekte mit eigenem Repertoire überhaupt finanzieren zu können.

In Ausnahmefällen ermöglicht das Tributekonzept auch höhere Einkünfte. Die Australier Björn Again absolvierten allein von 1989 bis 1993 über 800 meist ausverkaufte Konzerte mit ihrem ABBA-Programm. Höhepunkt war ein fünftägiges Engagement im Melbourner Town \& Country Club, das der Band eine Gage von 200.000 australischen Dollar einbrachte (Wells 1993). Auch die von dem Berliner Werner Leonard initiierten, international besetzten ABBA MANIA gehen weltweit auf Tournee. Sie schafften es, laut Leonard (Interview vom 7.11.2005), die KölnArena an zwei aufeinander folgenden Abenden zu füllen (20.000 Zuschauer) und spielten von der ebenfalls ausverkauften Berliner Waldbühne bis hin zum Kreml in Moskau. Das Jahreseinkommen einiger amerikanischer Tributebands wird auf 500.000 US-Dollar geschätzt (Hwang 2000).

Die deutsche Tributeszene kann von derlei Umsätzen wohl nur träumen - sieht man von einigen professionell gemanagten ABBA- oder Queen-Shows einmal ab. Die meisten Bands arbeiten als Amateure oder bestenfalls im semiprofessionellen Bereich. Im Normalfall dürfte der Spaß am Tun und die Begeisterung für die gedoubelten Interpreten im Vordergrund stehen. Einige zufällig ausgewählte Beispiele für die Häufigkeit der Auftritte, die den Homepages der jeweiligen Bands entnommen wurden (Zugriff: 11.10.2005), zeigen folgendes Bild:

- Die Beatles Revival Band absolvierte 2005 insgesamt 19 Auftritte.

- Die Stuttgarter Collins-Imitatoren Phil brachten es von Mitte Oktober bis Dezember 2005 auf sechs Verpflichtungen.

- Interstellar Overdrive, eine Pink-Floyd-Show aus Wiesbaden, spielte von Juni bis Oktober 2005 insgesamt viermal live. Darunter waren zwei Open Airs und ein Hallen-Rock-Festival.

- ABBA99 brachte es im Jahr 2005 immerhin auf 49 Auftritte.

Zwar liegen die Gagen deutscher Tributebands meist deutlich über denen von Coverbands oder Formationen mit eigenen Stücken und sind nicht selten im Bereich von 2.500 Euro oder darüber angesiedelt (Interview mit Kreis und Schütz vom 29.12.2005). Oftmals sind die Kosten, die Tributeshows verursachen, aber auch höher als bei anderen Bands. So benötigen KISSIN' TIME allein zwischen 150 und 250 Euro pro Auftritt für die eingesetzte Pyrotechnik, bei größeren Auftrittsorten entsprechend mehr: »Ein Teil der Gage wird 
direkt auf der Bühne in die Luft gesprengt« (ebd.). Hinzu kommen die Ausgaben für Maske, Kostümierung und den immer wieder zu aktualisierenden Instrumentenbestand. Auch ist der Markt für das Produkt Tributeshow immer mehr umkämpft. So berichten Kreis und Schütz von professionellen Bands aus Osteuropa, die mit zum Teil aggressivem Marketing und Preisdumping lokale Bands um Auftrittsmöglichkeiten bringen.

\section{Ausblick}

Dreißig Jahre nach den ersten Elvis- und Beatles-Imitatoren hat sich das Konzept Tributeband stilübergreifend zu einem scheinbar risikofreien Unterhaltungssegment mit Erfolgsgarantie etabliert. In einem Interview bezeichnet der Popkulturforscher Robert J. Thompson Tributebands kritisch als »lebende Museen populärer Musik« (zit. n. Kanaracus 2001). Folgt man den Äußerungen von Beteiligten, so lässt sich mancher Tributeshow allerdings ein fast schon didaktischer Charakter unterstellen: Laut Leonard (Interview vom 7.11.2005) bringen Konzerte von Formationen wie ABBA GOLD und ABBA MANIA den Siebziger-Jahre-Sound und die »für die damalige Zeit schon hochintellektuelle Musik« der Schweden auch einem jüngeren Publikum näher. Ähnlich äußert sich auch Rob Koritz von der GreatefulDead-Tributeband Dark Star: "Younger folks who never got to see the Dead get a taste of the music, the flavor of it « (zit. n. Tarnach 2002).

Schattenseite des Erfolgskonzepts Tributeshow ist die Negation eigener künstlerische Originalität. Antriebsfeder kann es sein, die Songs der jeweiligen Lieblingsband »zu zelebrieren und gemeinsam mit anderen Fans eine Party zu feiern « - wobei der Grad der angestrebten Authentizität mitunter von Publikum ernster genommen wird als von den Musikern selbst (Interview mit Kreis und Schütz vom 29.12.2005). Für viele Musiker, insbesondere in Nordamerika und Australien, ist die Mitgliedschaft in einer Tributeband jedoch weit entfernt von solcher Partystimmung, ist sie doch oftmals »the only way to make a living playing music (Kanaracus 2001).

Nicht alle (Nachwuchs-)Musiker mögen jedoch darauf verzichten, eigene Musikstücke zu präsentieren (Witzel 2000; Hemming 2002; Menzel 2005; Rosenbrock 2006), auch wenn mit der zunehmenden Nachfrage nach bloßer Reproduktion von Bekanntem, bereits Vorgelebtem ein Rückgang von Auftrittsmöglichkeiten für Bands mit eigenem Repertoire einhergeht. Auch goutieren nicht alle Fans von Original-Bands das Kopieren ihrer Favoriten. So fordert ein Zuhörer einer sich den finnischen Gothic-Rockern HIM widmenden Tributeband diese in ihrem Gästebuch (www.himself666.de; 
Zugriff: 30.12 .2005$)$ ultimativ auf: »Macht eigene Lieder mit euren eigenen Gefühlen und Emotionen!«

\section{Literatur}

Boucher, Geoff (2001). "The Long and Winding Road: Tribute bands that have labored to keep alive the myths made by other musicians are now getting recognition of their own. «In: The Los Angeles Times, 10. November, S. F.1.

Dambeck, Holger (2005). "Coverband Beatallica: Sony stoppt Metallica-Beatles. «In: Spiegel Online, 23. Februar, www.spiegel.de/netzwelt/netzkultur/0,1518, 343268,00.html (Zugriff: 5.10.2005).

Geary, Krissi (2005). »Tribute Bands: Flattering Imitators or Flagrant Infringers. « In: Southern Illinois University Law Journal, Vol. 29 (Spring), S. 481-507.

Groebbels, Udo (2004). „Gunz 'n' Rozes: Axl Rose spricht fließend holländisch. «In: Soundbase Online Musikmagazin, soundbase-online.com/ index.php3?br=0\&rub= intarchiv\&id=403 (Zugriff: 3.10.2004).

Hemming, Jan (2002). Begabung und Selbstkonzept. Eine qualitative Studie unter semi-professionellen Musikern in Rock und Pop. Münster u.a.: LIT.

Hwang, Suein L. (2000). "Loony Tunes? All the New Money Seems to Have a Thing For All the Old Music - And in San Francisco, That's Giving Real Bands Cause To Sing the IPO Blues - Why the Cheeseballs Rule.«In: The Wall Street Journal, 2. März, S. A1.

Kanaracus, Chris (2000). "I wanna be a rock-n-roll clone: Cashing in on covers: The enduring popularity of tribute bands." In: The Worcester Phoenix, 4.-11. Februar, www.worcesterphoenix.com/archive/features/00/02/04/TRIBUTE.html (Zugriff: 6.10.2005).

Kanaracus, Chris (2001). Cover me: Tribute bands may be campy and derivative, but they transport nostalgic fans to a happier time. «In: PROVIDENCE PHOENIX, 26. Oktober, www.providencephoenix.com/archive/features/01/10/25/cover. html (Zugriff: 12.9.2004).

Lehner, Christian (1999). "Nicht nur sein Pseudonym ist außergewöhnlich: der BRUZZLER. « In: Music Magazine, Nr. 45 (Dezember), www. bruzzler.com/xact. htm (Zugriff: 22.10.2004).

Menzel, Karl (2005). PC-Musiker - Der Einsatz computergestützter RecordingSysteme im Amateursektor. Osnabrück: epOs.

Morrow, Guy (2005). Selling Out or Buying In? (Unveröffentlichtes Manuskript).

Pendzich, Marc (2005). »Bühne frei für Blender. Revivalbands ahmen die legendären Gruppen vergangener Tage nach. Selten erreichen sie deren Klasse.« In: Rheinischer Merkur, 24. Februar, S. 21.

Rosenbrock, Anja (2006). Komposition in Pop- und Rockbands. Eine qualitative Studie zu kreativen Gruppenprozessen. Münster u.a.: LIT.

Roth, Wolf-Dieter (2005). „Copyright im Sony-Stil: Website einer Parodie-Musikgruppe gesperrt. "In: heise online, 4. März, www.heise.de/tp/r4/artikel/19/ 19593/1.html (Zugriff: 3.10.2005).

Ritchie, Jason (2003). »Rising stars: Van Hielen.«In: Get Ready To Rock. The Website vor Classic, Progressive \& Metal Rock, www.getreadytorock.com/rising stars/van_hielan.htm (Zugriff: 13.10.2004).

Rubinowsky, Leslie (1997). Impersonating Elvis. Boston: Faber and Faber. 
Simons, David (2001). "SEND IN THE CLONES: Tribute bands - gigging in the land of make believe.«In: Onstage, Oktober, onstagemag.com/ar/performance_send clones_tribute (Zugriff: 3.10 .2005$)$.

Tarlach, Gemma (2002). »Highway to History: Tribute Bands Rock Out Salutes to the Likes of AC/DC, KISS. « In: Journal Sentinel, 29. August (ohne Seitenangabe).

Wells, Ken (1993). »Maybe These Bands Will Start a Magazine Called Rolling Clone Exploiters of Rock's Fake Fad Look, Sound Like Big Stars But Charge Humbler Prices. «In: The Wall Street Journal, 5. Januar, S. A1.

Witzel, Thomas G. (2000). "Der musikalische Arbeitsprozess von Amateurbands. Eine empirische Untersuchung im Gießener Raum. «In: Populäre Musik im kulturwissenschaftlichen Diskurs. Hg. v. Helmut Rösing und Thomas Phleps (= Beiträge zur Popularmusikforschung, Bd. 25/26). Karben: CODA, S. 73-90.

Würfl, Matthias (2004). »Pink Floyd haargenau nachspielen.«In: Hessische Allgemeine (Sonntagszeitung), 18. April, S. 16.

\title{
Filme
}

Dokumentarfilme:

Economy, Jeff / Hacker, Darren (2000). ...An Incredible Simulation.

Fox, Richard / Curry, Kris (2001). Tribute - a Rockumentary.

Spielfilm:

Herek, Stephen (2001). Rock Star.

\section{Interviews}

Kreis, Matthias / Schütz, Thorsten (KISS-Tributeband KISSIN' TIME, Kassel), Interview mit dem Autor am 29.12.2005.

Leonard, Werner (Fidelio Entertainment Berlin, Initiator u.a. von ABBA Gold und ABBA MANIA), Interview mit dem Autor am 7.11.2005.

\begin{abstract}
Since the 1970s tribute band events have been a growing section of the entertainment market. Meanwhile nearly every successful rock or pop act has a variety of tribute bands trying to imitate the original artists' music and live performances. This article provides insight into the international tribute scene, into the practices of »tributing «, and informs about financial aspects of tribute shows.
\end{abstract}

